\title{
Universal quantization of the magnetic susceptibility jump at a topological phase transition
}

\author{
Soshun Ozaki $\oplus^{*}$ and Masao Ogata $(1)$ \\ Department of Physics, University of Tokyo, Bunkyo, Tokyo 113-0033, Japan
}

(Received 21 April 2020; accepted 16 November 2020; published 19 January 2021)

\begin{abstract}
We examine the magnetic susceptibility of topological insulators microscopically and find that the orbitalZeeman (OZ) cross term, the cross term between the orbital effect and the spin Zeeman effect, is directly related to the Berry curvature when the $z$ component of spin is conserved. In particular, the $\mathrm{OZ}$ cross term reflects the spin Chern number, which results in the quantization of the magnetic susceptibility jump at the topological phase transition. The magnitude of the jump is in units of the universal value $4|e| \mu_{B} / h$. We also apply the obtained formula to an explicit model and demonstrate the quantization. For this model, the physical origin of this quantization is clarified.
\end{abstract}

DOI: 10.1103/PhysRevResearch.3.013058

\section{INTRODUCTION}

Topological insulators (TIs) [1-13] show anomalous phenomena such as electric conduction on sample surfaces. Experimentally, the search for candidate materials for TIs is one of the most important problems. In particular, twodimensional (2D) TIs are predicted to show unique phenomena, such as the spin Hall effect and robust edge states against nonmagnetic impurities, only a few of which have been found [7-11]. So far, the confirmation of topological materials has been achieved by finding the edge state by angle-resolved photoemission spectroscopy or from the transport coefficients. Since both methods detect anomalous electronic states at the edge, it is desirable to develop some bulk-sensitive methods that enable us to confirm the topological nature of a material. In this paper, we propose that the quantization of the bulk magnetic susceptibility jump can be used as strong evidence for the topological phase transition in 2D TIs.

Usually, the magnetic susceptibility is discussed in terms of the orbital effect of the magnetic field [14-30] and spin Zeeman effect independently. In general, however, there can be a cross term between the orbital and Zeeman effects [5,6,31-35], which we call the orbital-Zeeman (OZ) cross term $\chi_{\mathrm{OZ}}$ in the following. Recently, Nakai and Nomura [34] discussed the jump in $\chi_{\mathrm{OZ}}$ at the topological phase transition using the formula of the orbital magnetization [36-42] and the Středa formula [43]. They calculated the OZ cross term in the Bernevig-Hughes-Zhang model [4] and concluded that the width of the jump depends on the $g$ factors of the involved orbitals introduced phenomenologically. In general, spin-orbit interaction (SOI) modifies the $g$ factor from its bare value

\footnotetext{
*ozaki@ hosi.phys.s.u-tokyo.ac.jp

Published by the American Physical Society under the terms of the Creative Commons Attribution 4.0 International license. Further distribution of this work must maintain attribution to the author(s) and the published article's title, journal citation, and DOI.
}

$g_{0}=2$. (Here we neglect the relativistic correction of $g_{0}$.) Thus, their conclusion means that the jump in $\chi_{\mathrm{OZ}}$ is not quantized in a universal value.

In the present paper, we study $\chi_{\mathrm{OZ}}$ microscopically based on the Green's function formalism and show that, in contrast to the results of Nakai and Nomura, the jump in $\chi_{\mathrm{Oz}}$ is exactly quantized in units of the universal value $4|e| \mu_{B} / h$ [see Eq. (9) below] even in the presence of SOI as long as the $z$ component of the spin is conserved. Here $\mu_{B}=|e| \hbar / 2 m$ is the Bohr magneton. When we study a model microscopically [as in Eq. (1)], the modification of the $g$ factor does not occur explicitly, and instead the effect of SOI appears in the deformation of the Bloch wave functions and the energy dispersion, which eventually leads to the orbital-dependent $g$ factors. We show below that the effect of SOI is exactly canceled out in $\chi_{\mathrm{Oz}}$, which leads to the quantization of jump with a universal value. In the latter part of this paper, we apply the obtained formula to a low-energy effective model for a topological insulator to show the validity of the present proposal. For this model, we clarify the physical origin of the quantization: It turns out that the quantization is associated with the chiral edge current, which is characteristic of the topologically nontrivial state.

\section{GENERAL FORMALISM}

First, we develop microscopically a general formula for magnetic susceptibility including orbital magnetism, Pauli paramagnetism, and the $\mathrm{OZ}$ cross term in terms of thermal Green's functions in the presence of SOI. Let $H$ be the general Hamiltonian derived from the Dirac equation in the presence of a general 3D periodic potential $V(\boldsymbol{r})$ and a magnetic field, which is given by

$$
\begin{aligned}
H= & \frac{1}{2 m}[\boldsymbol{p}-e \boldsymbol{A}(\boldsymbol{r})]^{2}-\frac{e \hbar}{2 m} \boldsymbol{\sigma} \cdot \boldsymbol{B}(\boldsymbol{r})+V(\boldsymbol{r}) \\
& +\frac{\hbar^{2}}{8 m^{2} c^{2}} \nabla^{2} V+\frac{\hbar}{4 m^{2} c^{2}} \boldsymbol{\sigma} \cdot \nabla V \times[\boldsymbol{p}-e \boldsymbol{A}(\boldsymbol{r})],
\end{aligned}
$$

where $\boldsymbol{A}(\boldsymbol{r})$ is a vector potential, $e<0$ for electrons, $\sigma=$ $\left(\sigma_{x}, \sigma_{y}, \sigma_{z}\right)$ are $2 \times 2$ Pauli matrices, and $\boldsymbol{B}(\boldsymbol{r})=\nabla \times \boldsymbol{A}(\boldsymbol{r})$ 
represents a magnetic field. The last term represents the SOI. It is to be noted that the second term representing the Zeeman interaction has the bare value $g_{0}=2$. As performed by Fukuyama [20], we implement a perturbative calculation of the free energy in terms of the vector potential $\boldsymbol{A}(\boldsymbol{r})$ via the Luttinger-Kohn representation [44]. As a result, we obtain the expression for each contribution as follows (the details of the derivation are shown in the Appendix):

$$
\begin{aligned}
\chi_{\text {orbit }} & =\frac{e^{2}}{2 \hbar^{2}} \frac{k_{B} T}{L^{3}} \sum_{n \boldsymbol{k}} \operatorname{Tr} \gamma_{x} \mathcal{G} \gamma_{y} \mathcal{G} \gamma_{x} \mathcal{G} \gamma_{y} \mathcal{G}, \\
\chi_{\text {Pauli }} & =-\frac{k_{B} T}{L^{3}} \sum_{n \boldsymbol{k}} \operatorname{Tr} M_{z}^{s} \mathcal{G} M_{z}^{s} \mathcal{G}, \\
\chi_{\mathrm{OZ}} & =-\frac{i|e|}{\hbar} \frac{k_{B} T}{L^{3}} \sum_{n \boldsymbol{k}} \operatorname{Tr}\left[M_{z}^{s} \mathcal{G} \gamma_{x} \mathcal{G} \gamma_{y} \mathcal{G}-M_{z}^{s} \mathcal{G} \gamma_{y} \mathcal{G} \gamma_{x} \mathcal{G}\right]
\end{aligned}
$$

where $\mathcal{G}$ is the thermal Green's function $\mathcal{G}\left(\boldsymbol{k}, i \varepsilon_{n}\right)$, whose $\left(l l^{\prime}\right)$ component is the matrix element between the $l$ th and $l^{\prime}$ th bands. Each band index includes the pseudospin degrees of freedom in the case with SOI. $\varepsilon_{n}$ is the Matsubara frequency, $\gamma_{\mu}$ represents the current operator in the $\mu$ direction divided by $e / \hbar$, and $M_{z}^{s}$ is the matrix for the operator $-\mu_{B} \sigma_{z}$. The effect of SOI is included in $\mathcal{G}$ and $\gamma_{\mu}$. Tr is the trace over the band indices and the spin degrees of freedom. In Eq. (2), $\chi_{\text {orbit }}$ and $\chi_{\text {Pauli }}$ represent the orbital and Pauli magnetic susceptibility, respectively. These are the same expressions as were obtained before $[20,45]$ even in the presence of SOI. On the other hand, $\chi_{\mathrm{OZ}}$ is the $\mathrm{OZ}$ cross term, which we focus on in this paper.

\section{UNIVERSAL QUANTIZATION OF $\chi_{\mathrm{OZ}}$}

In the following, we consider the case where the $z$ component of spin is conserved even in the presence of SOI. This situation will be realized more easily in $2 \mathrm{D}$ systems than in $3 \mathrm{D}$ systems. Therefore, we focus on $2 \mathrm{D}$ systems in the following. As shown in Appendix, it is straightforward to extend Eq. (2) in 2D systems where $V(\boldsymbol{r})$ gives a confinement potential in the $z$ direction and the magnetic field perpendicular to the 2D $x-y$ plane. Eventually, we obtain the formula for $2 \mathrm{D}$ systems by substituting the 3D $\boldsymbol{k}$ summation for a $2 \mathrm{D} \boldsymbol{k}$ summation and by including trace over the subband index for the $z$ direction wave function. Hereafter, we use bold for two-component vectors, e.g., $\boldsymbol{k}=\left(k_{x}, k_{y}\right)$.

Then, to discuss the quantization, we rewrite Eq. (2c) in terms of the Bloch wave functions in a similar way to Ref. [22]. The periodic part of the Bloch wave function $\hat{u}_{l k}(\boldsymbol{r}, z)={ }^{t}\left(u_{l k \uparrow}(\boldsymbol{r}, z), u_{l k \downarrow}(\boldsymbol{r}, z)\right)$ satisfies

$$
H_{k} \hat{u}_{l k}(\boldsymbol{r}, z)=\varepsilon_{l}(\boldsymbol{k}) \hat{u}_{l k}(\boldsymbol{r}, z), \quad H_{k}=e^{-i k r} H e^{i k r},
$$

where $l$ represents both the band index in the 2D Brillouin zone and the subband index in the $z$ direction. In the following, we abbreviate $u_{l \boldsymbol{k} \sigma}(\boldsymbol{r}, z)$ as $u_{l \boldsymbol{k} \sigma}$. When the SOI is given by $\left(\hbar / 4 m^{2} c^{2}\right) \sigma_{z}\left[\left(\partial_{x} V\right)\left(p_{y}-e A_{y}\right)-\left(\partial_{y} V\right)\left(p_{x}-e A_{x}\right)\right]$, the $z$ component of spin is conserved. In this case, up- and down-spin electrons are independent, and $u_{l k \uparrow}$ and $u_{l k \downarrow}$ are the eigenstates of $\sigma_{z}$ with eigenvalues $\varepsilon_{l \uparrow}(\boldsymbol{k})$ and $\varepsilon_{l \downarrow}(\boldsymbol{k})$ (denoted as $\varepsilon_{l \uparrow}$ and $\varepsilon_{l \downarrow}$ in the following). In this case, the matrices $\mathcal{G}$ and $M_{z}^{s}$ are diagonal and given by $\left[\mathcal{G}_{\sigma}\right]_{l l^{\prime}}=\delta_{l l^{\prime}}\left(i \varepsilon_{n}-\varepsilon_{l \sigma}+\mu\right)^{-1}$ and

$$
\begin{aligned}
{\left[M_{z}^{s}\right]_{l \sigma, l^{\prime} \sigma^{\prime}} } & =\int u_{l \boldsymbol{k} \sigma}^{*}\left(-\mu_{B} \sigma_{z}\right) u_{l^{\prime} \boldsymbol{k} \sigma^{\prime}} d \boldsymbol{r} d z \\
& =-\mu_{B} \sigma \delta_{l l^{\prime}} \delta_{\sigma \sigma^{\prime}},
\end{aligned}
$$

respectively. Note that the normalization is fixed by the equation

$$
\int u_{l \boldsymbol{k} \sigma}^{*} u_{l^{\prime} \boldsymbol{k} \sigma^{\prime}} d \boldsymbol{r} d z=\delta_{l l^{\prime}} \delta_{\sigma \sigma^{\prime}}
$$

where the spatial integral is over the entire crystal. On the other hand, the matrix $\gamma_{\mu}$ has off-diagonal matrix elements between the different bands and it becomes [20,46],

$$
\begin{aligned}
{\left[\gamma_{\mu \sigma}\right]_{l l^{\prime}} } & =\int u_{l \boldsymbol{k} \sigma}^{*} \frac{\partial H_{\boldsymbol{k}}}{\partial k_{\mu}} u_{l^{\prime} \boldsymbol{k} \sigma} d \boldsymbol{r} d z \\
& =\frac{\partial \varepsilon_{l \sigma}}{\partial k_{\mu}} \delta_{l l^{\prime}}+\left(\varepsilon_{l^{\prime} \sigma}-\varepsilon_{l \sigma}\right) \int u_{l \boldsymbol{k} \sigma}^{*} \frac{\partial u_{l^{\prime} \boldsymbol{k} \sigma}}{\partial k_{\mu}} d \boldsymbol{r} d z .
\end{aligned}
$$

Substituting these quantities into Eq. (2c) and carrying out the Matsubara summation, we obtain

$$
\chi_{\mathrm{OZ}}=-\frac{2|e| \mu_{B}}{\hbar L^{2}} \sum_{l \boldsymbol{k} \sigma} f\left(\varepsilon_{l \sigma}\right) \sigma \Omega_{l \boldsymbol{k} \sigma}^{z}+\frac{i|e| \mu_{B}}{\hbar L^{2}} \sum_{l \boldsymbol{k} \sigma} \sigma f^{\prime}\left(\varepsilon_{l}\right)\left\{\int \frac{\partial u_{l k \sigma}^{*}}{\partial k_{x}}\left(\varepsilon_{l \sigma}-H_{k}\right) \frac{\partial u_{l k \sigma}}{\partial k_{y}} d \boldsymbol{r} d z-(x \leftrightarrow y)\right\},
$$

where $\Omega_{l \sigma}^{z}$ is the Berry curvature in the $z$ direction,

$$
\Omega_{l \boldsymbol{k} \sigma}^{z}=i \int\left(\frac{\partial u_{l k \sigma}^{*}}{\partial k_{x}} \frac{\partial u_{l k \sigma}}{\partial k_{y}}-\frac{\partial u_{l k \sigma}^{*}}{\partial k_{y}} \frac{\partial u_{l k \sigma}}{\partial k_{x}}\right) d \boldsymbol{r} d z
$$

$f(\varepsilon)=\left[1+e^{(\varepsilon-\mu) / k_{B} T}\right]^{-1}$, and the completeness condition $\sum_{l^{\prime} \sigma} u_{l^{\prime} \boldsymbol{k} \sigma}(\boldsymbol{r}, z) u_{l^{\prime} \boldsymbol{k} \sigma}^{*}\left(\boldsymbol{r}^{\prime}, z^{\prime}\right)=\delta\left(\boldsymbol{r}-\boldsymbol{r}^{\prime}\right) \delta\left(z-z^{\prime}\right)$ has been used to take the summation over the intermediate state $l^{\prime}$. Especially for $2 \mathrm{D}$ insulator at zero temperature, the second term in Eq. (7) vanishes because the Fermi surface is absent. Then
$\chi_{\mathrm{OZ}}$ is written as

$$
\chi_{\mathrm{OZ}}^{2 \mathrm{D}}=-\frac{4|e| \mu_{B}}{h} \sum_{l: \mathrm{occ}} \mathrm{Ch}_{\mathrm{s}, l},
$$

where the summation $\sum_{l \text { :occ }}$ is taken for the occupied bands and $\mathrm{Ch}_{\mathrm{s}, l}$ is the spin Chern number for the $l$ th band defined by

$$
\mathrm{Ch}_{\mathrm{s}, l}=\frac{1}{2} \frac{2 \pi}{L^{2}} \sum_{k}\left(\Omega_{l k \uparrow}-\Omega_{l k \downarrow}\right) .
$$

At a topological phase transition, $\mathrm{Ch}_{\mathrm{s}, l}$ changes from one integer to another. Therefore, Eq. (9) leads to the quantization of the magnetic susceptibility jump at the topological 
phase transition. The magnitude of the jump is in units of $\chi_{0}=4|e| \mu_{B} / h$, which is universal. Although the effect of SOI is included in $u_{l k \sigma}$, it does not affect the coefficient in Eq. (9) since $\mathrm{Ch}_{\mathrm{s}, l}$ is a topological number, which leads to the universal quantization of jump in $\chi_{\mathrm{Oz}}$.

\section{EXPLICIT CALCULATION OF $\chi_{o z}$ IN A TOPOLOGICAL INSULATOR}

In the rest of this paper, we consider the low-energy effective model of the Kane-Mele model [1,2,47-51], one of the models for $2 \mathrm{D}$ TIs, to show that $\chi_{\mathrm{Oz}}$ actually has a jump at the topological phase transition and that other contributions do not conceal the quantized jump. We introduce the Kane-Mele model,

$$
\begin{aligned}
H=- & \sum_{\langle i, j\rangle \alpha} t c_{i \alpha}^{\dagger} c_{j \alpha}+\Delta_{0}\left(\sum_{i \in \mathrm{A}, \alpha} c_{i \alpha}^{\dagger} c_{i \alpha}-\sum_{i \in B, \alpha} c_{i \alpha}^{\dagger} c_{i \alpha}\right) \\
& +\sum_{\langle\langle i, j\rangle\rangle, \alpha \beta} i t_{2} v_{i j} c_{i \alpha}^{\dagger} s_{\alpha \beta}^{z} c_{j \beta},
\end{aligned}
$$

where $c_{i \alpha}^{\dagger}$ is the creation operator of an electron with spin $\alpha$ at site $i$, and the summation $\langle i, j\rangle(\langle\langle i, j\rangle\rangle)$ runs over all the nearest- (next-nearest-) neighbor sites of the 2D honeycomb lattice. The first term represents the usual nearest-neighbor hopping with transfer integral $t$. The second term represents a staggered on-site potential, $+\Delta_{0}$ for A sublattice and $-\Delta_{0}$ for $\mathrm{B}$ sublattice. The last term represents the hopping originating from SOI. We take account of only the $s^{z}$ component as in Ref. [2] and set $v_{i j}=-v_{j i}=+1(-1)$ if the electron makes a left (right) turn to propagate to the next-nearest sites. This model is known as one for silicene [47-51]. We can control $\Delta_{0}$ by changing the electric field applied perpendicular to the layer due to its buckled structure.

Performing the Fourier transform, we obtain the Hamiltonian in a matrix form,

$$
H_{k \sigma}=\left(\begin{array}{cc}
\Delta_{0}+\sigma \lambda_{k} & -t \gamma_{k}^{*} \\
-t \gamma_{k} & -\Delta_{0}-\sigma \lambda_{k}
\end{array}\right),
$$

where $\sigma=1$ is for up-spin and $\sigma=-1$ is for down-spin. The complex factor $\gamma_{k}$,

$$
\gamma_{\boldsymbol{k}}=e^{-i k_{y} a}+e^{i\left(\frac{\sqrt{3}}{2} k_{x}+\frac{1}{2} k_{y}\right) a}+e^{i\left(-\frac{\sqrt{3}}{2} k_{x}+\frac{1}{2} k_{y}\right) a},
$$

comes from the Fourier transform of hopping with $a$ being the distance between the nearest-neighbor sites, and

$$
\lambda_{k}=2 t_{2} \sin \frac{\sqrt{3}}{2} k_{x} a\left(\cos \frac{3}{2} k_{y} a-\cos \frac{\sqrt{3}}{2} k_{x} a\right)
$$

comes from the spin-dependent hopping, $t_{2}$. The energy dispersion of this model is given by

$$
E_{\sigma k}^{ \pm}= \pm \sqrt{\left(\Delta_{0}+\sigma \lambda_{k}\right)^{2}+t^{2}\left|\gamma_{k}\right|^{2}},
$$

which is shown in Fig. 1(a) with $\Delta_{0} / t=1 / 4, t_{2} / t=\sqrt{3} / 36$ (topologically trivial; solid line) and in Fig. 1(b) with $\Delta_{0} / t=$ $1 / 4, t_{2} / t=\sqrt{3} / 12$ (topologically nontrivial; dashed line). In the following, we use Figs. 1(a) and 1(b) as typical cases. Since the space inversion symmetry is broken, the energy dispersions for up and down spins can be different. In the

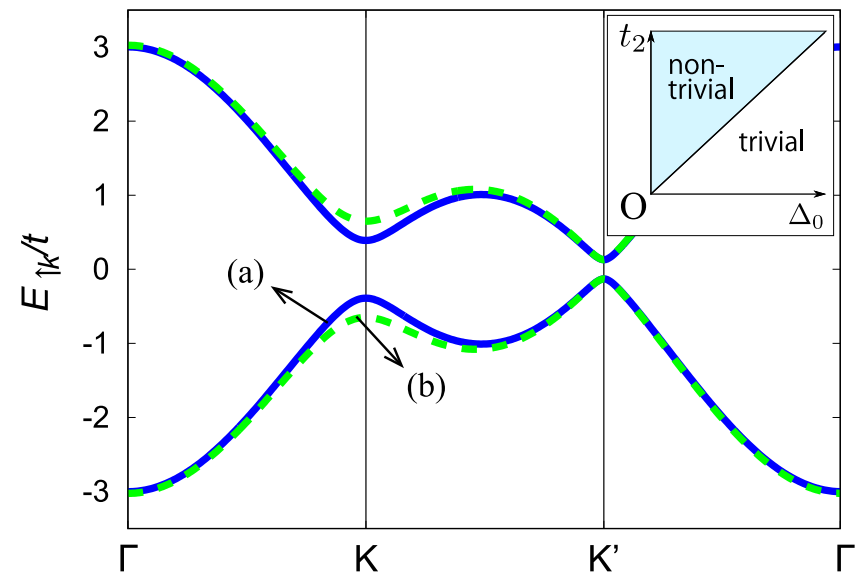

FIG. 1. Energy dispersion for $\sigma=1$ (up spin) of the model in Eq. (11) along the path $\Gamma \rightarrow K \rightarrow K^{\prime} \rightarrow \Gamma$ for two typical choices of parameters: (a) solid line, $\Delta_{0} / t=1 / 4, t_{2} / t=\sqrt{3} / 36$ (topologically trivial), and (b) dashed line, $\Delta_{0} / t=1 / 4, t_{2} / t=\sqrt{3} / 12$ (topologically nontrivial). The energy dispersion for $\sigma=-1$ (down spin) is obtained by exchanging $K$ for $K^{\prime}$ points. Inset: Phase diagram of this model.

momentum space, gaps open at $K=(4 \pi / 3 \sqrt{3} a, 0)$ and $K^{\prime}=$ $(-4 \pi / 3 \sqrt{3} a, 0)$. Their magnitudes are $2\left|\Delta_{0}+(3 \sqrt{3} / 2) \sigma t_{2}\right|$ at $K$ and $2\left|\Delta_{0}-(3 \sqrt{3} / 2) \sigma t_{2}\right|$ at $K^{\prime}$, respectively.

In this model, the ratio of $\Delta_{0}$ to $t_{2}$ determines the topological order [49-51]: topologically trivial for $\left|t_{2} / \Delta_{0}\right|<2 / 3 \sqrt{3}$ and topologically nontrivial for $\left|t_{2} / \Delta_{0}\right|>2 / 3 \sqrt{3}$. The phase diagram is shown in the inset of Fig. 1.

Before calculating magnetic susceptibility, let us examine the Berry curvature. Figure 2 shows the distribution of the Berry curvature in the momentum space for the valence band electrons with up spin for the two choices of the parameters in Fig. 1. It is seen that the Berry curvature is localized near $K$ and $K^{\prime}$ points. After numerical integration, we find that the Chern numbers are 0 for Fig. 2(a) and 1 for Fig. 2(b), which is consistent with the fact that Figs. 2(a) and 2(b) belong to the topologically trivial and nontrivial phases, respectively. According to Figs. 1 and 2, the low-energy excitations in the vicinity of $K$ and $K^{\prime}$ points are important when $\mu \simeq 0$. Therefore, we approximate the Hamiltonian by the expansion (a)

trivial

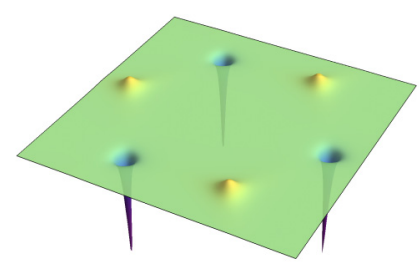

(b)

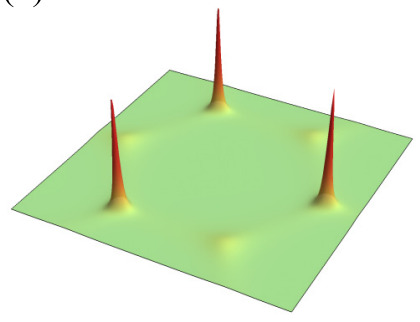

nontrivial
FIG. 2. Distribution of Berry curvature in the momentum space for the valence band electrons with up spin for case of (a) (topologically trivial) and (b) (topologically nontrivial). The Chern numbers are 0 for (a) and 1 for (b). 


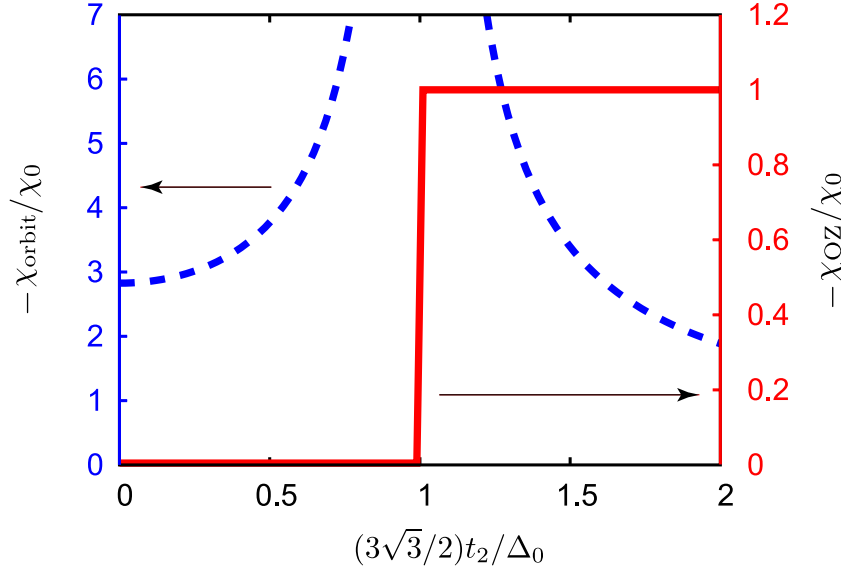

FIG. 3. Contributions to magnetic susceptibility as a function of $(3 \sqrt{3} / 2) t_{2} / \Delta_{0}$ for $\mu=0$ with $\Delta_{0}=t / 4$. They are normalized by $\chi_{0}$. The values of $v_{F}$ and $a$ are chosen to be the same as those of graphene. The system is topologically trivial (nontrivial) in the region $(3 \sqrt{3} / 2) t_{2} / \Delta_{0}<1(>1)$.

around $K$ and $K^{\prime}$ points, i.e., $\boldsymbol{k} \cdot \boldsymbol{p}$ perturbation. In this way, we obtain a low-energy effective model,

$$
h_{\sigma}^{K / K^{\prime}}=\left(\Delta_{\sigma}^{K / K^{\prime}}-\sigma \alpha k^{2}\right) \tau_{z}+\hbar v_{F} k_{x} \tau_{x}+\hbar v_{F} k_{y} \tau_{y},
$$

where $\tau_{x}, \tau_{y}$, and $\tau_{z}$ are the Pauli matrices representing the degrees of freedom of sublattices $\mathrm{A}$ and $\mathrm{B}, \Delta_{\sigma}^{K}=\Delta_{0}+$ $\sigma(3 \sqrt{3} / 2) t_{2}, \Delta_{\sigma}^{K^{\prime}}=-\Delta_{0}+\sigma(3 \sqrt{3} / 2) t_{2}, \alpha=(9 \sqrt{3} / 8) a^{2} t_{2}$, and $\hbar v_{F}=(3 / 2) a t$. Note that the signs of the mass term $\Delta_{\sigma}^{K / K^{\prime}}$ at $K$ and $K^{\prime}$ points with different spins are opposite, i.e., $\Delta_{\uparrow}^{K}=-\Delta_{\downarrow}^{K^{\prime}}$ and $\Delta_{\downarrow}^{K}=-\Delta_{\uparrow}^{K^{\prime}}$. This effective Hamiltonian is justified in the limit of $t_{2}, \Delta_{0} \rightarrow 0$ with $t_{2} / \Delta_{0}$ fixed.

Let us discuss the magnetic susceptibility. Note that Ezawa [50] calculated the orbital magnetism but the $\mathrm{OZ}$ cross term was not taken into account. In the model Eq. (16), the thermal Green's function is defined as $\mathcal{G}_{\sigma}^{K / K^{\prime}}=\left(i \varepsilon_{n}-h_{\sigma}^{K / K^{\prime}}\right)^{-1}$. The current operator in the $\mu$ direction is given by $\gamma_{\mu}=$ $\hbar v_{F} \tau_{\mu}-2 \sigma \alpha k_{\mu} \tau_{z}$. Substituting these quantities into Eq. (2), carrying out the Matsubara summation, and performing the 2D momentum integration at $T=0$, we obtain

$$
\begin{aligned}
\chi_{\text {orbit }} & =-\frac{e^{2} v_{F}^{2}}{6 \pi} \sum_{\eta=K, K^{\prime}} \frac{1}{\left|\Delta_{\uparrow}^{\eta}\right|} \theta\left(\left|\Delta_{\uparrow}^{\eta}\right|-|\mu|\right), \\
\chi_{\text {Pauli }} & =\frac{\mu_{B}^{2}}{\pi \hbar^{2} v_{F}^{2}} \sum_{\eta=K, K^{\prime}}|\mu| \theta\left(|\mu|-\left|\Delta_{\uparrow}^{\eta}\right|\right), \\
\chi_{\mathrm{OZ}} & =-\frac{2 \mu_{B}|e|}{h} \sum_{\eta=K, K^{\prime}} \operatorname{sgn}\left(\Delta_{\uparrow}^{\eta}\right) \theta\left(\left|\Delta_{\uparrow}^{\eta}\right|-|\mu|\right),
\end{aligned}
$$

in the limit of $t_{2}, \Delta_{0} \rightarrow 0$ with $t_{2} / \Delta_{0}$ fixed. Here $\chi_{\text {orbit }}$ is the orbital diamagnetic susceptibility of the 2D Dirac electrons discussed in the preceding studies [23-29]. $\chi_{\text {Pauli }}$ is the Pauli paramagnetism proportional to the density of states $(\propto|\mu|)$.

To observe the quantization of the jump, we focus on the case of $\mu=0$, an insulating case, where $\chi_{\text {Pauli vanishes. }}$ Figure 3 shows $\chi_{\text {orbit }}$ and $\chi_{\mathrm{OZ}}$ as a function of $t_{2} / \Delta_{0}$. When $(3 \sqrt{3} / 2) t_{2} / \Delta_{0}<1$, the system is topologically trivial and the signs of $\Delta_{\uparrow}^{K}$ and $\Delta_{\uparrow}^{K^{\prime}}$ are opposite, which leads to $\chi_{\mathrm{OZ}}=0$ (a)

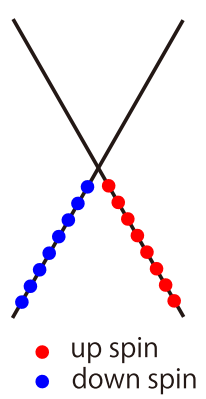

(b)

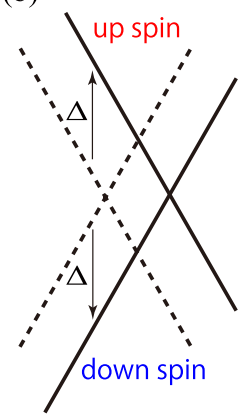

(c)

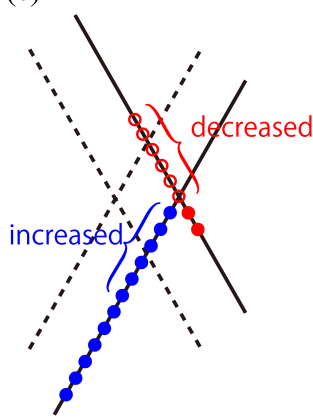

FIG. 4. Schematic pictures for the edge state. (a) Ground state without an external magnetic field. (b) Change caused by the Zeeman interaction. (c) New ground state in the magnetic field.

from Eq. (17c). When $(3 \sqrt{3} / 2) t_{2} / \Delta_{0}>1$, on the other hand, the system is topologically nontrivial and the signs of $\Delta_{\uparrow}^{K}$ and $\Delta_{\uparrow}^{K^{\prime}}$ are the same, which leads to $\chi_{\mathrm{oz}}=-\chi_{0}$. As a result, $\chi_{\mathrm{OZ}}$ has a universal jump at the topological phase transition at $t_{2} / \Delta_{0}=2 / 3 \sqrt{3}$. On the other hand, $\chi_{\text {orbit }}$ diverges at the phase transition due to the gap closing. However, we can see that $\chi_{\text {orbit }}=-\frac{e^{2} v_{F}^{2}}{6 \pi}\left(\left|\Delta_{0}-3 \sqrt{3} t_{2} / 2\right|^{-1}+\left|\Delta_{0}+3 \sqrt{3} t_{2} / 2\right|^{-1}\right)$ and the magnitude of divergence is the same on both sides of the phase transition. Therefore, when we subtract the divergence of $\chi_{\text {orbit }}$, we will be able to detect the jump in $\chi_{\mathrm{oz}}$. Note that the effect of SOI represented by $t_{2}$ appears only in the magnitude of $\chi_{\text {orbit }}$ and does not affect the magnitude of

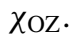

\section{PHYSICAL ORIGIN OF QUANTIZATION}

Let us consider the physical origin of the quantization. $\chi_{\mathrm{OZ}}$ is interpreted as the sum of the correction to the orbital magnetic moment induced by the magnetic field that couples to the spin magnetic moment and the correction to the spin magnetic moment induced by the magnetic field that couples to the orbital magnetic moment. Here we estimate the former correction. In the edge state, there are spin-polarized linear dispersions and a spin current flows. Figure 4(a) corresponds to the state with $\mathrm{Ch}_{\mathrm{s}, l}=+1$. (Note that the number of pairs of dispersion coincides with $\left|\mathrm{Ch}_{\mathrm{s}, l}\right|$.) When a magnetic field is applied through the Zeeman interaction $\mu_{B} \sigma_{z} B$, the upspin (down-spin) band moves upward (downward) as shown in Fig. 4(b). The width of change $\Delta$ is $\mu_{B} B$. Then, in the lowest energy state [Fig. 4(c)], the number of down-spin (up-spin) electrons increases (decreases) by $v \mu_{B} B$, where $v$ is the density of states $v=L / c h$ and $c$ is the velocity of the edge current. This change leads to an electric current of $-2|e| \mu_{B} B / h$ in the right direction, which causes the orbital magnetic moment of $-2|e| \mu_{B} B / h$ per area. The coefficient of $B$ is half of the quantization of magnetic susceptibility, $-\chi_{0} / 2$. We can also estimate the contribution from the other correction (i.e., the spin magnetic moment induced by an energy shift originating from an orbital magnetic moment made by a circular electric current), which gives the same value. Combining these two contributions, we obtain the $\mathrm{OZ}$ cross term as $-\chi_{0}$, which is consistent with Eq. (9). 


\section{DISCUSSION AND CONCLUSION}

The expression for the magnetic susceptibility including the spin Zeeman effect was obtained in terms of Bloch wave functions [35], which contains a term $\chi_{\text {occ: } 2}=$ $-\frac{e}{\hbar} \operatorname{Re} \sum_{l k} f\left(\varepsilon_{k}\right) M_{l l}^{z} \Omega_{l}^{z}$. Actually, $\chi_{\mathrm{occ}: 2}$ gives half of $\chi_{\mathrm{Oz}}$ in Eq. (9). The origin of this difference is as follows. In Ref. [35], the effect of Zeeman interaction is distributed among several terms for total magnetic susceptibility including $\chi_{\text {occ: } 2}$. Therefore, if we collect all the effects of Zeeman interaction in the formalism of Ref. [35], then we can recover $\chi_{\mathrm{Oz}}$.

Based on the microscopic theory, we have derived a new simple formula for magnetic susceptibility in a Bloch system with SOI and Zeeman interaction to show that the OZ cross term, one of the three contributions to magnetic susceptibility, is always quantized in units of the universal value $4|e| \mu_{B} / h$ for $2 \mathrm{D}$ spin-conserving insulators at zero temperature. We have also applied the formula to a model for a 2D TI and demonstrated the quantization. For this model, we have clarified that this quantization originates from the redistribution of the chiral edge state due to the magnetic field. We expect that this discussion is also qualitatively applicable to other 2D TIs. Our results clearly show that the magnetic response reflects the topological nature of a material. Therefore, it should be possible to make a bulk-sensitive confirmation of the topological phase transition.

\section{ACKNOWLEDGMENTS}

We thank H. Matsuura, H. Maebashi, I. Tateishi, T. Hirosawa, N. Okuma, and V. Könye for very fruitful discussions. This work was supported by Grants-in-Aid for Scientific Research from the Japan Society for the Promotion of Science (Grant No. JP18H01162). S.O. was supported by the Japan Society for the Promotion of Science through the Program for Leading Graduate Schools (MERIT).

\section{APPENDIX: DERIVATION OF THE MAGNETIC SUSCEPTIBILITY FORMULA FOR PERIODIC SYSTEMS}

\section{3D cases}

We consider Bloch electrons in a $3 \mathrm{D}$ periodic potential $V(\boldsymbol{r})$, Zeeman interaction, and SOI. For this purpose, we use the following Hamiltonian derived from the Dirac equation in the presence of a magnetic field:

$$
\begin{aligned}
H= & \frac{1}{2 m}[\boldsymbol{p}-e \boldsymbol{A}(\boldsymbol{r})]^{2}-\frac{e \hbar}{2 m} \boldsymbol{\sigma} \cdot \boldsymbol{B}(\boldsymbol{r})+V(\boldsymbol{r}) \\
& +\frac{\hbar^{2}}{8 m^{2} c^{2}} \nabla^{2} V+\frac{\hbar}{4 m^{2} c^{2}} \boldsymbol{\sigma} \cdot \nabla V \times[\boldsymbol{p}-e \boldsymbol{A}(\boldsymbol{r})],
\end{aligned}
$$

where $\boldsymbol{A}(\boldsymbol{r})$ is a vector potential, $e<0$ for electrons, and $\boldsymbol{B}(\boldsymbol{r})=\nabla \times \boldsymbol{A}(\boldsymbol{r})$ represents a magnetic field. The $g$ factor for electrons is assumed to be $g=2$.

The magnetic susceptibility per volume is defined as

$$
\chi=-\frac{1}{L^{3}} \lim _{B \rightarrow 0} \frac{\partial^{2} \Omega}{\partial B^{2}},
$$

where $\Omega$ is the thermodynamic potential of the system with chemical potential $\mu$, i.e.,

$$
\Omega=-k_{B} T \ln \operatorname{Tr} \exp [-\beta(H-\mu N)] .
$$

Therefore, it is sufficient to evaluate the second-order deviation of $\Omega$ due to a magnetic field, defined as $\Omega^{(2)}$.

To perform the pertubative calculations with respect to $A$ and $\boldsymbol{B}$ as was done by Fukuyama [20], we rewrite the Hamiltonian in a second quantized form as

$$
H=H_{0}+H_{1}+H_{2}
$$

with

$$
\begin{aligned}
& H_{0}=\sum_{\alpha \beta} \int \psi_{\alpha}^{\dagger}(\boldsymbol{r})\left\{-\frac{\hbar^{2}}{2 m} \nabla^{2}+V(\boldsymbol{r})+\frac{\hbar^{2}}{8 m^{2} c^{2}} \nabla^{2} V(\boldsymbol{r})+\frac{\hbar}{4 m^{2} c^{2}} \sigma_{\alpha \beta} \cdot \nabla V \times \boldsymbol{p}\right\} \psi_{\beta}(\boldsymbol{r}) d \boldsymbol{r} \\
& H_{1}=-\int \boldsymbol{j}(\boldsymbol{r}) \cdot \boldsymbol{A}(\boldsymbol{r}) d \boldsymbol{r}-\sum_{\alpha \beta} \int \psi_{\alpha}^{\dagger}(\boldsymbol{r}) \frac{e \hbar}{2 m} \boldsymbol{\sigma}_{\alpha \beta} \cdot \boldsymbol{B}(\boldsymbol{r}) \psi_{\beta}(\boldsymbol{r}) d \boldsymbol{r} \\
& H_{2}=\sum_{\alpha} \frac{e^{2}}{2 m} \int \boldsymbol{A}^{2}(\boldsymbol{r}) \psi_{\alpha}^{\dagger}(\boldsymbol{r}) \psi_{\beta}(\boldsymbol{r}) d \boldsymbol{r},
\end{aligned}
$$

where $\boldsymbol{j}(\boldsymbol{r})$ is the current operator without a vector potential,

$$
\begin{aligned}
\boldsymbol{j}= & \frac{e}{2 m} \sum_{\alpha}\left\{\psi_{\alpha}^{\dagger}\left(\boldsymbol{p} \psi_{\alpha}\right)-\left(\boldsymbol{p} \psi_{\alpha}^{\dagger}\right) \psi_{\alpha}\right\} \\
& +\frac{e \hbar}{4 m^{2} c^{2}} \sum_{\alpha \beta} \psi_{\alpha}^{\dagger} \boldsymbol{\sigma}_{\alpha \beta} \times \nabla V \psi_{\beta} .
\end{aligned}
$$

The subscript of each Hamiltonian represents the order of $\boldsymbol{A}$. To avoid unphysical contributions due to the unbounded character of the coordinate operators, we introduce the vector potential $\boldsymbol{A}$ with Fourier component $\boldsymbol{q}$, as was done by
Hebborn and Sondheimer [16],

$$
\boldsymbol{A}(\boldsymbol{r})=-i \boldsymbol{A}_{\boldsymbol{q}}\left(e^{i \boldsymbol{q} \boldsymbol{r}}-e^{-i \boldsymbol{q} \boldsymbol{r}}\right),
$$

and let $\boldsymbol{q}=0$ in the final expressions.

To make the pertubative calculation easier, we employ the Luttinger-Kohn representation [44],

$$
\psi_{\alpha}(\boldsymbol{r})=\sum_{l \boldsymbol{k} \alpha} \chi_{l \boldsymbol{k} \alpha}(\boldsymbol{r}) a_{l k \alpha}, \quad \chi_{l k \alpha}(\boldsymbol{r})=e^{i k \boldsymbol{r}} u_{l k_{0} \alpha}(\boldsymbol{r}),
$$

where $\boldsymbol{k}_{0}$ is a fixed wave vector and $\hat{u}_{l \boldsymbol{k}}(\boldsymbol{r})={ }^{t}\left(u_{l \boldsymbol{k} \uparrow}(\boldsymbol{r}), u_{l \boldsymbol{k} \downarrow}(\boldsymbol{r})\right)$ is the periodic part of the Bloch wave function with the wave 
vector $k$ of the $l$ th band. $\hat{u}_{l k}$ satisfies

$$
H_{k} \hat{u}_{l k}(\boldsymbol{r})=\varepsilon_{l k} \hat{u}_{l k}(\boldsymbol{r})
$$

with

$$
\begin{aligned}
H_{k}= & \frac{\hbar^{2}}{2 m}(\boldsymbol{k}-i \nabla)^{2}+V(\boldsymbol{r})+\frac{\hbar^{2}}{8 m^{2} c^{2}} \nabla^{2} V \\
& +\frac{\hbar^{2}}{4 m^{2} c^{2}} \boldsymbol{\sigma} \cdot \nabla V \times(\boldsymbol{k}-i \nabla) .
\end{aligned}
$$

In this representation, $H_{0}, H_{1}$, and $H_{2}$ are rewritten as

$$
\begin{aligned}
H_{0}= & \sum_{l l^{\prime} \alpha \beta \boldsymbol{k}} E_{l l^{\prime} \alpha \beta} a_{l \boldsymbol{k} \alpha}^{\dagger} a_{l^{\prime} \boldsymbol{k} \beta}, \\
H_{1}= & \frac{i e}{\hbar} \boldsymbol{A}_{\boldsymbol{q}} \cdot \sum_{l l^{\prime} \alpha \beta \boldsymbol{k}} \boldsymbol{\gamma}(\boldsymbol{k})\left(a_{l \boldsymbol{k}+\frac{q}{2} \alpha}^{\dagger} a_{l^{\prime} \boldsymbol{k}-\frac{q}{2} \beta}-a_{l \boldsymbol{k}-\frac{q}{2} \alpha}^{\dagger} a_{l^{\prime} \boldsymbol{k}+\frac{q}{2} \beta}\right) \\
& -\boldsymbol{q} \times \boldsymbol{A}_{\boldsymbol{q}} \cdot \sum_{l l^{\prime} \alpha \beta \boldsymbol{k}} \boldsymbol{M}^{S}\left(a_{l \boldsymbol{k}+\frac{q}{2} \alpha}^{\dagger} a_{l^{\prime} \boldsymbol{k}-\frac{q}{2} \beta}+a_{l \boldsymbol{k}-\frac{q}{2} \alpha}^{\dagger} a_{l^{\prime} \boldsymbol{k}+\frac{q}{2} \beta}\right), \\
H_{2}= & -\frac{e^{2}}{2 m} \sum_{\alpha} \boldsymbol{A}_{\boldsymbol{q}}^{2}\left(\rho_{-2 \boldsymbol{q} \alpha}-2 \rho_{0 \alpha}+\rho_{2 \boldsymbol{q} \alpha}\right),
\end{aligned}
$$

where

$$
\begin{aligned}
E_{l l^{\prime} \alpha \beta} & =\int u_{l \boldsymbol{k}_{0} \alpha}^{*} H_{\boldsymbol{k}} u_{l^{\prime} \boldsymbol{k}_{0} \beta} d \boldsymbol{r}, \\
{[\gamma(\boldsymbol{k})]_{l \alpha, l^{\prime} \beta} } & =\int u_{l \boldsymbol{k}_{0} \alpha}^{*} \frac{\partial H_{k}}{\partial \boldsymbol{k}} u_{l^{\prime} \boldsymbol{k}_{0} \beta} d \boldsymbol{r}, \\
{\left[\boldsymbol{M}^{s}\right]_{l \alpha, l^{\prime} \beta} } & =\int u_{l \boldsymbol{k}_{0} \alpha}^{*} \frac{e \hbar}{2 m} \sigma u_{l^{\prime} \boldsymbol{k}_{0} \beta} d \boldsymbol{r}, \\
\rho_{\boldsymbol{q}} & =\sum_{l \boldsymbol{k} \alpha} a_{l \boldsymbol{k}-\boldsymbol{q} \alpha}^{\dagger} a_{l \boldsymbol{k} \alpha} .
\end{aligned}
$$

By using the Luttinger-Kohn representation, the thermodynamic potential becomes

$$
\Omega=\Omega_{0}+k_{B} T \int_{0}^{1} \frac{d \lambda}{\lambda} \sum_{\boldsymbol{k} n} \operatorname{Tr}\left[\Sigma^{\star \lambda}\left(\boldsymbol{k}, i \varepsilon_{n}\right) \mathcal{G}^{\lambda}\left(\boldsymbol{k}, i \varepsilon_{n}\right)\right],
$$

where $\Omega_{0}$ is the thermodynamic potential without a vector potential. $\Sigma^{\star \lambda}$ and $\mathcal{G}^{\lambda}$ are the self-energy matrix and thermal Green's function corresponding to the Hamiltonian $H_{0}+$ $\lambda\left(H_{1}+H_{2}\right)$, respectively. We expand the self-energy as

$$
\Sigma^{\star \lambda}=\lambda \Sigma^{\star(1)}+\lambda^{2} \Sigma^{\star(2)}+O\left(A^{3}\right) .
$$

Making use of Dyson's equation,

$$
\mathcal{G}^{\lambda}\left(\boldsymbol{k}, i \varepsilon_{n}\right)=\mathcal{G}^{(0)}\left(\boldsymbol{k}, i \varepsilon_{n}\right)+\mathcal{G}^{(0)}\left(\boldsymbol{k}, i \varepsilon_{n}\right) \Sigma^{\star \lambda}\left(\boldsymbol{k}, i \varepsilon_{n}\right) \mathcal{G}^{\lambda}\left(\boldsymbol{k}, i \varepsilon_{n}\right),
$$

with $\mathcal{G}^{(0)}$ being Green's function for the nonperturbative Hamiltonian $H_{0}$, we can carry out the integral as

$$
\Omega-\Omega_{0}=k_{B} T \sum_{k n} \operatorname{Tr}\left[\Sigma^{\star(1)} \mathcal{G}^{(0)}+\frac{1}{2} \Sigma^{\star(2)} \mathcal{G}^{(0)}\right]+O\left(A^{3}\right) .
$$

To estimate Eq. (A19), we find nine types of contributions of the order of $\boldsymbol{A}^{2}$, whose diagrams are shown in Fig. 5. Figures 5(1a)-5(1c), 5(2a)-5(2d), and 5(3a) and 5(3b) give the orbital contribution, the orbital-Zeeman cross term contribution, and the Zeeman contribution to the free energy, respectively. Each contribution is calculated as

$$
\begin{aligned}
\Omega_{\text {orbit }}^{(2)}= & \frac{1}{2} k_{B} T \sum_{n \boldsymbol{k}} \frac{e^{2}}{\hbar^{2}} A_{\boldsymbol{q} \mu} A_{\boldsymbol{q} \nu} \operatorname{Tr}\left[\gamma_{\mu} \mathcal{G}\left(\boldsymbol{k}+\frac{\boldsymbol{q}}{2}, i \varepsilon_{n}\right) \gamma_{\nu} \mathcal{G}\left(\boldsymbol{k}-\frac{\boldsymbol{q}}{2}, i \varepsilon_{n}\right)+(\boldsymbol{q} \leftrightarrow-\boldsymbol{q})\right]+k_{B} T \sum_{n \boldsymbol{k}} \frac{e^{2}}{m} \boldsymbol{A}_{\boldsymbol{q}}^{2} \operatorname{Tr} \mathcal{G}\left(\boldsymbol{k}, i \varepsilon_{n}\right), \\
\Omega_{\mathrm{OZ}}^{(2)}= & -\frac{1}{2} k_{B} T \sum_{n \boldsymbol{k}} \operatorname{Tr}\left[\frac{i e}{\hbar} A_{\boldsymbol{q} \mu} \gamma_{\mu} \mathcal{G}\left(\boldsymbol{k}+\frac{\boldsymbol{q}}{2}, i \varepsilon_{n}\right) \boldsymbol{q} \times \boldsymbol{A}_{\boldsymbol{q}} \cdot \boldsymbol{M}^{s} \mathcal{G}\left(\boldsymbol{k}-\frac{\boldsymbol{q}}{2}, i \varepsilon\right)+(\boldsymbol{q} \leftrightarrow-\boldsymbol{q})\right] \\
& +\frac{1}{2} k_{B} T \sum_{n \boldsymbol{k}} \operatorname{Tr}\left[\boldsymbol{q} \times \boldsymbol{A}_{\boldsymbol{q}} \cdot \boldsymbol{M}^{s} \mathcal{G}\left(\boldsymbol{k}+\frac{\boldsymbol{q}}{2}, i \varepsilon_{n}\right) \frac{i e}{\hbar} A_{\boldsymbol{q} \mu} \gamma_{\mu} \mathcal{G}\left(\boldsymbol{k}-\frac{\boldsymbol{q}}{2}, i \varepsilon_{n}\right)+(\boldsymbol{q} \leftrightarrow-\boldsymbol{q})\right], \\
\Omega_{\text {Zeeman }}^{(2)}= & \frac{1}{2} k_{B} T \sum_{n \boldsymbol{k}} \operatorname{Tr}\left[\boldsymbol{q} \times \boldsymbol{A}_{\boldsymbol{q}} \cdot \boldsymbol{M}^{s} \mathcal{G}\left(\boldsymbol{k}+\frac{\boldsymbol{q}}{2}, i \varepsilon_{n}\right) \boldsymbol{q} \times \boldsymbol{A}_{\boldsymbol{q}} \cdot \boldsymbol{M}^{s} \mathcal{G}\left(\boldsymbol{k}-\frac{\boldsymbol{q}}{2}, i \varepsilon_{n}\right)+(\boldsymbol{q} \leftrightarrow-\boldsymbol{q})\right],
\end{aligned}
$$

respectively. By expanding the expression for small $\boldsymbol{q}$ with the help of the Ward identity,

$$
\mathcal{G}\left(\boldsymbol{k}, i \varepsilon_{n}\right) \gamma_{\mu} \mathcal{G}\left(\boldsymbol{k}, i \varepsilon_{n}\right)=\frac{\partial \mathcal{G}\left(\boldsymbol{k}, i \varepsilon_{n}\right)}{\partial k_{\mu}},
$$

we obtain

$$
\begin{aligned}
\Omega_{\text {orbit }}^{(2)} & =-\frac{e^{2}}{2 \hbar^{2}}\left(\boldsymbol{q} \times \boldsymbol{A}_{\boldsymbol{q}}\right)_{z}^{2} k_{B} T \sum_{n \boldsymbol{k}} \operatorname{Tr}\left[\gamma_{x} \mathcal{G} \gamma_{y} \mathcal{G} \gamma_{x} \mathcal{G} \gamma_{y} \mathcal{G}\right] \\
\Omega_{\mathrm{OZ}}^{(2)} & =-\frac{i e}{\hbar}\left(\boldsymbol{q} \times \boldsymbol{A}_{\boldsymbol{q}}\right)_{z}^{2} k_{B} T \sum_{n k} \operatorname{Tr}\left[M_{z}^{s} \mathcal{G} \gamma_{x} \mathcal{G} \gamma_{y} \mathcal{G}-M_{z}^{s} \mathcal{G} \gamma_{y} \mathcal{G} \gamma_{x} \mathcal{G}\right] \\
\Omega_{\text {Zeeman }}^{(2)} & =\left(\boldsymbol{q} \times \boldsymbol{A}_{\boldsymbol{q}}\right)_{z}^{2} k_{B} T \sum_{n k} \operatorname{Tr}\left[M_{z}^{s} \mathcal{G} M_{z}^{s} \mathcal{G}\right] .
\end{aligned}
$$



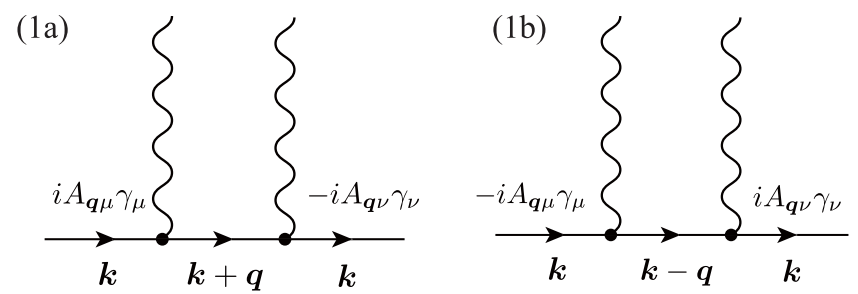

(1c)

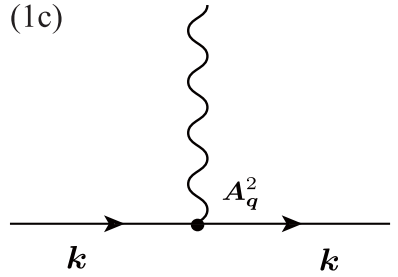

(2a)

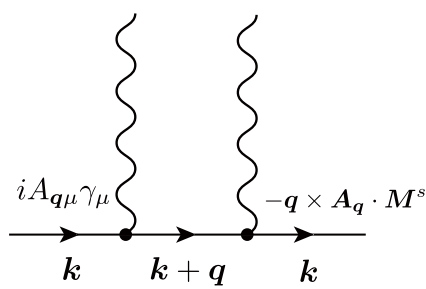

(2c)

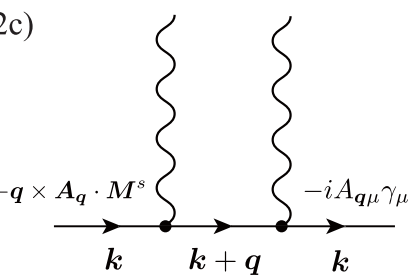

(3a)

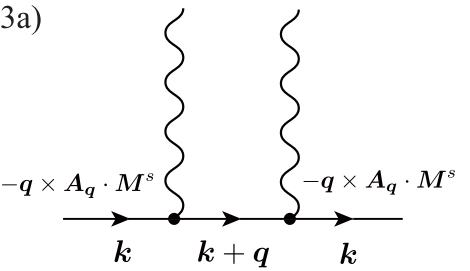

(2b)

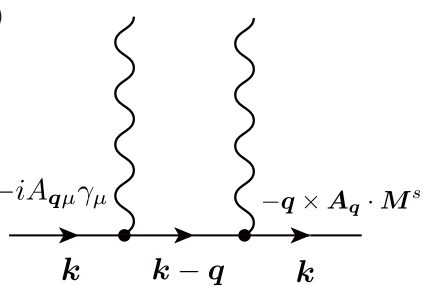

(2d)

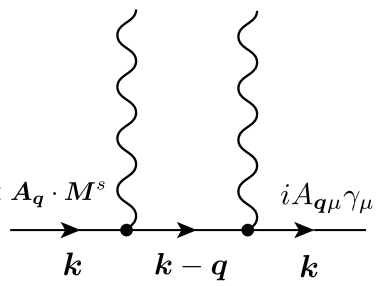

(3b)

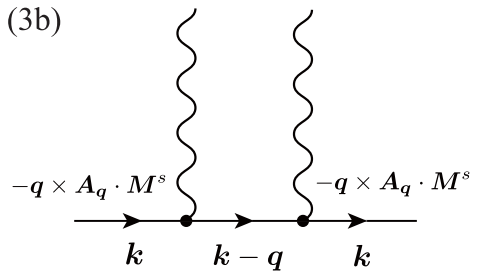

FIG. 5. Feynman diagrams for the second-order deviation of the free energy $\Omega^{(2)}$.

Since $2\left(\boldsymbol{q} \times \boldsymbol{A}_{\boldsymbol{q}}\right)_{z}^{2}$ corresponds to $B_{z}^{2}$, the magnetic susceptibility becomes

$$
\chi=\chi_{\text {orbit }}+\chi_{\mathrm{oz}}+\chi_{\text {Pauli }}
$$

where

$$
\begin{aligned}
& \chi_{\text {orbit }}=\frac{e^{2}}{2 \hbar^{2}} \frac{k_{B} T}{L^{3}} \sum_{n \boldsymbol{k}} \operatorname{Tr}\left[\gamma_{x} \mathcal{G} \gamma_{y} \mathcal{G} \gamma_{x} \mathcal{G} \gamma_{y} \mathcal{G}\right] \\
& \chi_{\mathrm{OZ}}=\frac{i e}{\hbar} \frac{k_{B} T}{L^{3}} \sum_{n \boldsymbol{k}} \operatorname{Tr}\left[M_{z}^{s} \mathcal{G} \gamma_{x} \mathcal{G} \gamma_{y} \mathcal{G}-M_{z}^{s} \mathcal{G} \gamma_{y} \mathcal{G} \gamma_{x} \mathcal{G}\right] \\
& \chi_{\text {Pauli }}=-\frac{k_{B} T}{L^{3}} \sum_{n \boldsymbol{k}} \operatorname{Tr}\left[M_{z}^{s} \mathcal{G} M_{z}^{s} \mathcal{G}\right] .
\end{aligned}
$$

Since the Luttinger-Kohn representation is associated with the Bloch representation by a unitary transformation [44], the expressions in Eq. (A26) can be regarded as written in terms of Green's functions and current operators for the Bloch representation.

\section{2D cases}

In the derivation in the previous section, we have assumed a $3 \mathrm{D}$ periodic potential. However, when the magnetic field is applied in the $z$ direction, the assumption of periodicity in the $z$ direction is unnecessary, as shown in this section. Therefore, the same discussion as in the $3 \mathrm{D}$ case is applicable to $2 \mathrm{D}$ systems, where electrons are confined in the $z$ direction.

We consider the case where the elctronic potential $V(x, y, z)$ is periodic in the $x-y$ plane perpendicular to the magnetic field. Hereafter, we use bold for two-component vectors, e.g., $\boldsymbol{k}=\left(k_{x}, k_{y}\right)$. From Bloch's theorem, the wave function is characterized by $\boldsymbol{k}$, which is written as

$$
\hat{\psi}_{k}(x, y, z)=e^{i k r} \hat{u}_{k}(x, y, z)
$$

where $\hat{u}_{k}(x, y, z)$ is a function which has the same periodicity as $V(x, y, z)$ in the $x-y$ plane. Since Eq. (A27) is the solution of the Schrödinger equation

$$
H \hat{\psi}_{k}(x, y, z)=E_{k} \hat{\psi}_{k}(x, y, z),
$$


$\hat{u}_{k}(x, y, z)$ satisfies the equation

$$
\begin{aligned}
& {\left[\frac{\hbar^{2}}{2 m}\left\{\left(k_{x}-i \frac{\partial}{\partial x}\right)^{2}+\left(k_{y}-i \frac{\partial}{\partial y}\right)^{2}-\frac{\partial^{2}}{\partial z^{2}}\right\}+V(x, y, z)\right.} \\
& \left.\quad+\frac{\hbar^{2}}{8 m^{2} c^{2}} \nabla^{2} V+\frac{\hbar^{2}}{4 m^{2} c^{2}} \sigma \cdot \nabla V \times\left(\begin{array}{c}
k_{x}-i \partial / \partial x \\
k_{y}-i \partial / \partial y \\
-i \partial / \partial z
\end{array}\right)\right] \hat{u}_{k}(x, y, z)=E_{k} \hat{u}_{k}(x, y, z) .
\end{aligned}
$$

Since the wave function $\hat{u}_{k}(x, y, z)$ is defined on a region $[0, L] \times[0, L] \times(-\infty, \infty)$ and is normalizable on the region, the energy eigenvalues take descrete values. When $V(x, y, z)$ gives a confinement potential in the $z$ direction, the wave functions are characterized by the band index in the $k_{x}-k_{y}$ Brillouin zone and the subband index in the $z$ direction. Therefore, we denote the wave function as $\hat{u}_{l k}(x, y, z)$, where $l$ represents the above two indices. Using $\hat{u}_{l k}$ 's, we can apply the same discussion as in the previous section and derive a similar formula. The Luttinger-Kohn representation is modified:

$$
\begin{aligned}
\psi_{\alpha}(x, y, z) & =\sum_{l k \alpha} \chi_{l k \alpha}(x, y, z) a_{l k \alpha}, \\
\chi_{l k \alpha}(x, y, z) & =e^{i k r} u_{l k_{0} \alpha}(x, y, z) .
\end{aligned}
$$

When the magnetic field is parallel to the $z$ direction, we can assume without loss of generarity that both the vector potential $\boldsymbol{A}_{\boldsymbol{q}}$ and $\boldsymbol{q}$ have only $x$ and $y$ components. Then, $H_{1}$ is expressed in this $2 \mathrm{D}$ scheme as

$$
\begin{aligned}
H_{1}= & \frac{i e}{\hbar} \boldsymbol{A}_{\boldsymbol{q}} \cdot \sum_{l l^{\prime} \alpha \beta \boldsymbol{k}} \boldsymbol{\gamma}(\boldsymbol{k})\left(a_{l \boldsymbol{k}+\frac{q}{2} \alpha}^{\dagger} a_{l^{\prime} \boldsymbol{k}-\frac{q}{2} \beta}-a_{l \boldsymbol{k}-\frac{q}{2} \alpha}^{\dagger} a_{l^{\prime} \boldsymbol{k}+\frac{q}{2} \beta}\right) \\
& -\left(q_{x} A_{\boldsymbol{q} y}-q_{y} A_{\boldsymbol{q} x}\right) \sum_{l l^{\prime} \alpha \beta \boldsymbol{k}} M_{z}^{s}\left(a_{l \boldsymbol{k}+\frac{q}{2} \alpha}^{\dagger} a_{l^{\prime} \boldsymbol{k}-\frac{q}{2} \beta}+a_{l \boldsymbol{k}-\frac{q}{2} \alpha}^{\dagger} a_{l^{\prime} \boldsymbol{k}+\frac{q}{2} \beta}\right),
\end{aligned}
$$

while $H_{0}$ and $H_{2}$ do not change formally. We note that the spatial integrals in matrix elements $E_{l l^{\prime} \alpha \beta}, \boldsymbol{\gamma}(\boldsymbol{k})$, and $M_{z}^{s}$ are $3 \mathrm{D}$ integrals. After all, we obtain a very similar formula

$$
\begin{aligned}
\chi_{\text {orbit }} & =\frac{e^{2}}{2 \hbar^{2}} \frac{k_{B} T}{L^{2}} \sum_{n \boldsymbol{k}} \operatorname{Tr}\left[\gamma_{x} \mathcal{G} \gamma_{y} \mathcal{G} \gamma_{x} \mathcal{G} \gamma_{y} \mathcal{G}\right] \\
\chi_{\mathrm{OZ}} & =\frac{i e}{\hbar} \frac{k_{B} T}{L^{2}} \sum_{n \boldsymbol{k}} \operatorname{Tr}\left[M_{z}^{s} \mathcal{G} \gamma_{x} \mathcal{G} \gamma_{y} \mathcal{G}-M_{z}^{s} \mathcal{G} \gamma_{y} \mathcal{G} \gamma_{x} \mathcal{G}\right] \\
\chi_{\text {Pauli }} & =-\frac{k_{B} T}{L^{2}} \sum_{n \boldsymbol{k}} \operatorname{Tr}\left[M_{z}^{s} \mathcal{G} M_{z}^{s} \mathcal{G}\right]
\end{aligned}
$$

where the value is normalized by $L^{2}$, different from the 3D case, and $\operatorname{Tr}$ is the trace over $l$ and $\alpha$.

[1] C. L. Kane and E. J. Mele, $Z_{2}$ Topological Order and the Quantum Spin Hall Effect, Phys. Rev. Lett. 95, 146802 (2005).

[2] C. L. Kane and E. J. Mele, Quantum Spin Hall Effect in Graphene, Phys. Rev. Lett. 95, 226801 (2005).

[3] B. A. Bernevig and S.-C. Zhang, Quantum Spin Hall Effect, Phys. Rev. Lett. 96, 106802 (2006).

[4] B. A. Bernevig, T. L. Hughes, and S.-C. Zhang, Quantum spin Hall effect and topological phase transition in HgTe quantum wells, Science 314, 1757 (2006).

[5] M.-F. Yang and M.-C. Chang, Středa-like formula in the spin Hall effect, Phys. Rev. B 73, 073304 (2006).

[6] S. Murakami, Quantum Spin Hall Effect and Enhanced Magnetic Response by Spin-Orbit Coupling, Phys. Rev. Lett. 97, 236805 (2006).

[7] M. König, S. Wiedmann, C. Brüne, A. Roth, H. Buhmann, L. W. Molenkamp, X.-L. Qi, and S.-C. Zhang, Quantum spin
Hall insulator state in HgTe quantum wells, Science 318, 766 (2007).

[8] A.Roth, C. Brüne, H. Buhmann, L. W. Molenkamp, J. Maciejko, X.-L. Qi, and S.-C. Zhang, Nonlocal transport in the quantum spin Hall state, Science 325, 294 (2009).

[9] C. Brüne, A. Roth, H. Buhmann, E. M. Hankiewicz, L. W. Molenkamp, J. Maciejko, X.-L. Qi, and S.-C. Zhang, Spin polarization of the quantum spin Hall edge states, Nat. Phys. 8, 485 (2012).

[10] I. Knez, R.-R. Du, and G. Sullivan, Evidence for Helical Edge Modes in Inverted InAs/GaSb Quantum Wells, Phys. Rev. Lett. 107, 136603 (2011).

[11] I. Knez, R.-R. Du, and G. Sullivan, Andreev Reflection of Helical Edge Modes in InAs/GaSb Quantum Spin Hall Insulator, Phys. Rev. Lett. 109, 186603 (2012). 
[12] L. Fu and C. L. Kane, Topological insulators with inversion symmetry, Phys. Rev. B 76, 045302 (2007).

[13] D. Hsieh, D. Qian, L. Wray, Y. Xia, Y. S. Hor, R. J. Cava, and M. Z. Hasan, A topological Dirac insulator in a quantum spin Hall phase, Nature 452, 970 (2008).

[14] L. D. Landau, Diamagnetismus der Metalle, Z. Phys. 64, 629 (1930).

[15] R. Peierls, Zur theorie des Diamagnetismus von Leitungselektronen, Z. Phys. 80, 763 (1933).

[16] J. Hebborn and E. Sondheimer, The diamagnetism of conduction electrons in metals, J. Phys. Chem. Solids 13, 105 (1960).

[17] E. I. Blount, Bloch electrons in a magnetic field, Phys. Rev. 126, 1636 (1962).

[18] J. Hebborn, J. Luttinger, E. Sondheimer, and P. Stiles, The orbital diamagnetic susceptibility of Bloch electrons, J. Phys. Chem. Solids 25, 741 (1964).

[19] G. H. Wannier and U. N. Upadhyaya, Zero-field susceptibility of Bloch electrons, Phys. Rev. 136, A803 (1964).

[20] H. Fukuyama, Theory of orbital magnetism of Bloch electrons: Coulomb interactions, Prog. Theor. Phys. 45, 704 (1971).

[21] H. Fukuyama and R. Kubo, Interband effects on magnetic susceptibility. II. Diamagnetism of bismuth, J. Phys. Soc. Jpn. 28, 570 (1970)

[22] M. Ogata and H. Fukuyama, Orbital magnetism of Bloch electrons I. General formula, J. Phys. Soc. Jpn. 84, 124708 (2015).

[23] J. W. McClure, Diamagnetism of graphite, Phys. Rev. 104, 666 (1956).

[24] H. Fukuyama, Anomalous orbital magnetism and Hall effect of massless fermions in two dimension, J. Phys. Soc. Jpn. 76, 043711 (2007).

[25] G. Gómez-Santos and T. Stauber, Measurable Lattice Effects on the Charge and Magnetic Response in Graphene, Phys. Rev. Lett. 106, 045504 (2011).

[26] M. Koshino and T. Ando, Anomalous orbital magnetism in Dirac-electron systems: Role of pseudospin paramagnetism, Phys. Rev. B 81, 195431 (2010).

[27] M. Ogata, Orbital magnetism of Bloch electrons: III. Application to graphene, J. Phys. Soc. Jpn. 85, 104708 (2016).

[28] Y. Gao, S. A. Yang, and Q. Niu, Geometrical effects in orbital magnetic susceptibility, Phys. Rev. B 91, 214405 (2015).

[29] A. Raoux, F. Piéchon, J.-N. Fuchs, and G. Montambaux, Orbital magnetism in coupled-bands models, Phys. Rev. B 91, 085120 (2015).

[30] F. Piéchon, A. Raoux, J.-N. Fuchs, and G. Montambaux, Geometric orbital susceptibility: Quantum metric without Berry curvature, Phys. Rev. B 94, 134423 (2016).

[31] N. Ito and K. Nomura, Anomalous Hall effect and spontaneous orbital magnetization in antiferromagnetic Weyl metal, J. Phys. Soc. Jpn. 86, 063703 (2017).

[32] Y. Tserkovnyak, D. A. Pesin, and D. Loss, Spin and orbital magnetic response on the surface of a topological insulator, Phys. Rev. B 91, 041121(R) (2015).
[33] M. Koshino and I. F. Hizbullah, Magnetic susceptibility in three-dimensional nodal semimetals, Phys. Rev. B 93, 045201 (2016).

[34] R. Nakai and K. Nomura, Crossed responses of spin and orbital magnetism in topological insulators, Phys. Rev. B 93, 214434 (2016).

[35] M. Ogata, Theory of magnetization in Bloch electron systems, J. Phys. Soc. Jpn. 86, 044713 (2017).

[36] D. Xiao, M.-C. Chang, and Q. Niu, Berry phase effects on electronic properties, Rev. Mod. Phys. 82, 1959 (2010).

[37] T. Thonhauser, Theory of orbital magnetization in solids, Int. J. Mod. Phys. B 25, 1429 (2011).

[38] G. Sundaram and Q. Niu, Wave-packet dynamics in slowly perturbed crystals: Gradient corrections and Berry-phase effects, Phys. Rev. B 59, 14915 (1999).

[39] D. Xiao, J. Shi, and Q. Niu, Berry Phase Correction to Electron Density of States in Solids, Phys. Rev. Lett. 95, 137204 (2005).

[40] T. Thonhauser, D. Ceresoli, D. Vanderbilt, and R. Resta, Orbital Magnetization in Periodic Insulators, Phys. Rev. Lett. 95 137205 (2005).

[41] D. Ceresoli, T. Thonhauser, D. Vanderbilt, and R. Resta, Orbital magnetization in crystalline solids: Multi-band insulators, Chern insulators, and metals, Phys. Rev. B 74, 024408 (2006).

[42] J. Shi, G. Vignale, D. Xiao, and Q. Niu, Quantum Theory of Orbital Magnetization and Its Generalization to Interacting Systems, Phys. Rev. Lett. 99, 197202 (2007).

[43] P. Středa, Theory of quantised Hall conductivity in two dimensions, J. Phys. C: Solid State Phys. 15, L717 (1982).

[44] J. M. Luttinger and W. Kohn, Motion of electrons and holes in perturbed periodic fields, Phys. Rev. 97, 869 (1955).

[45] N. Okuma and M. Ogata, Long-range Coulomb interaction effects on the surface Dirac electron system of a threedimensional topological insulator, J. Phys. Soc. Jpn. 84, 034710 (2015).

[46] A. H. Wilson, The Theory of Metals (Cambridge University Press, Cambridge, UK, 1953).

[47] G. G. Guzmán-Verri and L. C. Lew Yan Voon, Electronic structure of silicon-based nanostructures, Phys. Rev. B 76, 075131 (2007).

[48] C.-C. Liu, H. Jiang, and Y. Yao, Low-energy effective Hamiltonian involving spin-orbit coupling in silicene and twodimensional germanium and tin, Phys. Rev. B 84, 195430 (2011).

[49] M. Ezawa, A topological insulator and helical zero mode in silicene under an inhomogeneous electric field, New J. Phys. 14, 033003 (2012).

[50] M. Ezawa, Topological phase transition and electrically tunable diamagnetism in silicene, Eur. Phys. J. B 85, 363 (2012).

[51] C.-C. Liu, W. Feng, and Y. Yao, Quantum Spin Hall Effect in Silicene and Two-Dimensional Germanium, Phys. Rev. Lett. 107, 076802 (2011). 